\title{
AFFINE TRANSFORMATIONS AND ANALYTIC CAPACITIES
}

\author{
THOMAS DOWLING AND ANTHONY G. O'FARRELL
}

\begin{abstract}
Analytic capacities are set functions defined on the plane which may be used in the study of removable singularities, boundary smoothness and approximation of analytic functions belonging to some function space. The symmetric concrete Banach spaces form a class of function spaces that includes most spaces usually studied. The Beurling transform is a certain singular integral operator that has proved useful in analytic function theory. It is shown that the analytic capacity associated to each Beurling-invariant symmetric concrete Banach space behaves reasonably under affine transformation of the plane. It is not known how general analytic capacities behave under affine maps.
\end{abstract}

\section{The Beurling transform AND SYMMETRIC CONCRETE BANACH SPACES}

This paper is essentially self-contained, except that it assumes a knowledge of basic complex analysis, functional analysis, and distribution theory. We do make incidental use of a result from the Calderon-Zygmund theory, but this could easily be replaced by an alternative elementary but slightly more timeconsuming procedure.

1. We denote by $C^{\infty}$ the Fréchet algebra of all infinitely-differentiable complex-valued functions on the complex plane $C$, and by $C_{c s}^{\infty}$ the nuclear algebra of all complex-valued test functions on $\mathbf{C}$. The space of distributions on $\mathbf{C}$ is the dual $\mathrm{C}_{\mathrm{cs}}^{\infty \prime}$ of $\mathrm{C}_{\mathrm{cs}}^{\infty}$, and the space of distributions having compact support is the dual $\mathrm{C}^{\infty \prime}$ of $\mathrm{C}^{\infty}$. We use the notation $\langle\phi, f\rangle$ to denote the action of the linear functional $f$ on the element $\phi$. In the case where $f$ is (representable by) an integrable function, this means that

$$
\langle\phi, f\rangle=\int_{\mathbf{C}} \phi \cdot f d x d y .
$$

The Beurling transform $B$ is defined on test functions by

$$
(B \phi)(w)=-\frac{\mathbf{P V}}{\pi} \int_{\mathbf{C}} \frac{\phi(z) d x d y}{(z-w)^{2}}=-\frac{\mathbf{P V}}{\pi} \int_{\mathbf{C}} \frac{\phi(w-z) d x d y}{z^{2}}, \quad \forall \phi \in \mathbf{C}_{\mathrm{cs}}^{\infty} .
$$

$B$ maps $\mathrm{C}_{\mathrm{cs}}^{\infty}$ continuously into $\mathrm{C}^{\infty}$, and is symmetric in the sense that

$$
\int_{\mathbf{C}} \phi \cdot B \psi d x d y=\int_{\mathbf{C}} \psi \cdot B \phi d x d y, \quad \forall \phi, \psi \in \mathrm{C}_{\mathrm{cs}}^{\infty} .
$$

Received by the editors January 24, 1994 and, in revised form, October 5, 1994; originally communicated to the Proceedings of the AMS by Palle E. T. Jorgensen.

1991 Mathematics Subject Classification. Primary 30E99.

Key words and phrases. Analytic capacity, symmetric concrete space.

(C)1995 American Mathematical Society 
2. For a distribution $f$ having compact support, we define the distribution $B_{1} f$ by

$$
\left\langle\phi, B_{1} f\right\rangle=\langle B \phi, f\rangle, \quad \forall \phi \in \mathrm{C}_{\mathrm{cs}}^{\infty} .
$$

In other words, $B_{1}$ is the adjoint of $B$. Since $B$ is symmetric, it is clear that $B_{1}$ is an extension of $B$ from $C_{\mathrm{cs}}^{\infty}$ to $C^{\infty \prime}$. The map $B_{1}$ is continuous from $\mathrm{C}^{\infty \prime}$ into $\mathrm{C}_{\mathrm{cs}}^{\infty \prime}$.

3. The Beurling kernel $\frac{-1}{\pi z^{2}}$ is a Calderon-Zygmund cancellation kernel, and so the Calderon-Zygmund theory [cf. Stein 1970] shows that $B$ is a bounded map in $L^{p}$ norm whenever $1<p<+\infty$. In other words, for each $p \in$ $(1,+\infty)$, there exists $\kappa(p)>0$ such that

$$
\|B \phi\|_{L^{p}} \leq \kappa\|\phi\|_{L^{p}}, \quad \forall \phi \in \mathrm{C}_{\mathrm{cs}}^{\infty} .
$$

In particular, $B$ is bounded in $L^{3}$ norm. Also, $C_{\mathrm{cs}}^{\infty}$ is dense in $L^{3}$ in $L^{3}$ norm. This allows us to define a unique extension $B_{2}$ of $B$ to $L^{3}$ by requiring that

$$
B_{2} f=\lim B \phi_{n}
$$

(convergence in $L^{3}$ norm) whenever the $\phi_{n}$ belong to $\mathrm{C}_{\mathrm{cs}}^{\infty}$ and converge to $f$ in $L^{3}$ norm.

4. The two extensions of $B$ just defined agree on the overlap $L^{3} \cap C^{\infty /}$ of their respective domains:

Lemma. If $f \in \mathrm{C}^{\infty \prime}$ is (representable by integration against) an $L^{3}$ function, then $B_{1} f=B_{2} f$.

Proof. We may choose $\phi_{n} \in \mathrm{C}_{\mathrm{cs}}^{\infty}$ such that $\phi_{n} \rightarrow f$ in $L^{3}$ norm, and the supports of all the $\phi_{n}$ lie inside a fixed compact set. This is enough to ensure that the $\phi_{n}$ also converge to $f$ in the topology of $C^{\infty \prime}$. The continuity of $B_{1}$ and $B_{2}$ then yields

$$
B_{1} f=\lim B \phi_{n}=B_{2} f .
$$

Thus, since both maps are linear, we may define (unambiguously) a common extension $B_{3}$ on $L^{3}+C^{\infty \prime}$ by setting

$$
B_{3} f=B_{1} f_{1}+B_{2} f_{2}
$$

whenever $f=f_{1}+f_{2}$ with $f_{1} \in \mathrm{C}^{\infty \prime}$ and $f_{2} \in L^{3}$.

Henceforth we use $B$ to denote this extension $B_{3}$.

We abbreviate $L^{3}+C^{\infty \prime}$ to EL3 (mnemonic for Eventually $L^{3}$ ).

We remark that this is by no means a maximal reasonable extension of the Beurling transform. It is simply sufficient for our purposes. In fact, we only need to use the transform on a somewhat smaller space than EL3.

5. We denote by EAZI-the letters stand for Eventually Analytic and Zero at Infinity-the space of all distributions $f$ that are analytic outside some compact set and tend to zero at infinity.

In what follows we will have occasion to use the Beurling transform on functions of the form $f \circ T$, where $f \in \mathrm{EAZI}$ and $T$ is affine. It is easy to see that EAZI is a subspace of EL3, and that composition with affine functions maps EL3 into itself, so $B f$ is a well-defined distribution when $f$ is such a function.

6. A space $F$ of distributions equipped with some norm is a symmetric concrete Banach space (SCBS) if $F$ is complete and 
(1) the inclusions

are continuous,

$$
\mathrm{C}_{\mathrm{cs}}^{\infty} \hookrightarrow F \hookrightarrow \mathrm{C}_{\mathrm{cs}}^{\infty \prime}
$$

(2) $F$ is a topological $\mathrm{C}_{\mathrm{cs}}^{\infty}$-module with respect to pointwise multiplication,

(3) $\|\bar{f}\|_{F}=\|f\|_{F}, \forall f \in F$

and

(4) compact sets of invertible affine transformations of $\mathbf{C}$ act equicontinuously on $F$ by composition: whenever $K \subset$ Aff is a compact set of affine transformations, there exists a constant $\kappa>0$ such that the distributions $f \circ T$, defined by

satisfy

$$
\langle\phi, f \circ T\rangle=|T|^{-1}\left\langle\phi \circ T^{-1}, f\right\rangle, \quad \forall \phi \in \mathrm{C}_{\mathrm{cs}}^{\infty},
$$

$$
\|f \circ T\|_{F} \leq \kappa\|f\|_{F}, \quad \forall f \in F, \forall T \in K .
$$

7. We say that a symmetric concrete Banach space $F$ is $B$-invariant if there exists a constant $\kappa>0$ such that

$$
\|B f\|_{F} \leq \kappa\|f\|_{F}, \quad \forall f \in F \cap \text { EL3. }
$$

Notice that this is slightly cavalier, in that the unwary reader might suppose that if $F$ is $B$-invariant, then $F$ lies in the domain of $B$. In fact, if $F \cap E L 3$ is dense in $F$, then $B$ has a unique bounded extension to $F$, but otherwise this may or may not be the case. In practice, this is not a great abuse of language. The most important SCBS are the small ones and their duals-recall that an SCBS is small if $\mathrm{C}_{\mathrm{cs}}^{\infty}$ is dense in it. If $F$ is small, then obviously $F \cap \mathrm{EL} 3$ is dense in $F$. If $F=G^{*}$, where $F$ is $B$-invariant and $G$ is small, then $B$ has a unique weak-star continuous extension to $F$.

8. The Beurling transform $u=B f$ provides a solution to the partial differential equation

$$
\frac{\partial u}{\partial \bar{z}}=\frac{\partial f}{\partial z}
$$

It is related to the Cauchy transform, which is defined for test functions by

$$
(C \phi)(w)=\frac{1}{\pi} \int_{\mathbf{C}} \frac{\phi(z)}{z-w} d x d y
$$

and extended to $C^{\infty \prime}$ by duality (note that $C$ is skew-symmetric). We have

$$
B f=\frac{\partial C f}{\partial z} \text {. }
$$

The function $u=C f$ satisfies the partial differential equation

$$
\frac{\partial u}{\partial \bar{z}}=f
$$

\section{ANAlytic $F$-CAPACITIES AND STATEMENT OF MAIN RESUlt}

1. Let $F$ be a SCBS. We say that a distributrion $f$ is $\bar{\partial}-F$-admissible for the compact set $E \subset \mathbf{C}$ if $f \in F, f$ is analytic on $\mathbf{C} \sim E, f(\infty)=0$ and $\|f\|_{F} \leq 1$.

For such $f$, we denote by $f^{\prime}(\infty)$ the coefficient $a_{1}$ in the Laurent expansion

$$
f(z)=\frac{a_{1}}{z}+\frac{a_{2}}{z^{2}}+\cdots .
$$


The analytic capacity associated to $F$ is the set function $\bar{\partial}-F$-cap, defined on compact sets $E$ by

$$
\bar{\partial}-F \text {-cap }(E)=\sup \left\{\left|f^{\prime}(\infty)\right|: f \text { is } \bar{\partial}-F \text {-admissible for } E\right\} \text {. }
$$

The original analytic capacity introduced by Ahlfors is the $\bar{\partial}-L^{\infty}$-cap, and the continuous analytic capacity of Dolzhenko is the $\bar{\partial}-B C$-cap. Of other analytic capacities, the $\bar{\partial}$-Lip $\alpha$-cap, the $\bar{\partial}$-lip $\alpha$-cap, the $\bar{\partial}-L^{p}$-cap, and the $\bar{\partial}$-BMO-cap have been intensively studied.

Many analytic capacities have been constructively described up to bounded equivalence, but a number have not. In attempting to define the characteristics of a genuinely "real-variable" description, one of us (O'F) was led to consider the behaviour of analytic capacities under (real-)affine maps. It soon became apparent that this posed a nontrivial problem.

The question is this: Given an SCBS, $F$, a compact set $K \subset \mathbf{C}$, and a compact set $A$ of invertible affine maps, does there exist a constant $\kappa(F, K, A) \geq 1$ such that

$$
\frac{\bar{\partial}-F-\operatorname{cap}(E)}{\kappa} \leq \bar{\partial}-F-\operatorname{cap}(T E) \leq \kappa \cdot \bar{\partial}-F-\operatorname{cap}(E)
$$

whenever $E \subset K$ and $T \in A$ ? If $F$ has this property, then we say that its analytic capacity has the affine-equivalence property.

This question remains open in general. The purpose of the present paper is to give a partial positive result, as follows.

Theorem. Let $F$ be a Beurling-invariant symmetric concrete Banach space. Then its analytic capacity has the affine-equivalence property.

We will in fact find that if translation acts isometrically on $F$, then the constant $\kappa(F, K, A)$ may be taken of the form

$$
\kappa(F, K, A)=\kappa_{1}(F, K, \tau),
$$

where

$$
\tau=\sup \{\max \{\|T\|, 1 /\|T\|\}: T \in A\} .
$$

Here $\|T\|$ denotes the operator norm of the derivative of $T$. (By the operator norm of a linear function $L: \mathbf{R}^{2} \rightarrow \mathbf{R}^{2}$ we mean $\sup \{|L z|:|z| \leq 1\}$. We will use $|T|$ to denote the determinant of $D T$.)

The theorem covers all spaces $F$ that are locally well-behaved under the action of Calderon-Zygmund singular integral operators. This includes most wellknown spaces. Notorious exceptions include $L^{\infty}, C^{0}$, Lipl and the Sobolev space $W^{1,1}$. For these exceptions, we do not know whether or not the corresponding analytic capacity is affine-invariant.

The theorem is not very interesting except for spaces of locally-integrable functions. If $F$ properly contains $L_{\text {loc }}^{1}$, then singletons have positive capacity and the affine-equivalence holds trivially. There are of course SCBS that are unrelated to $L_{\text {loc }}^{1}$ by local inclusion (i.e. neither locally contain nor are locally contained in it), but none are well-known spaces. Accordingly, we will present the proof in two stages: first we will cover the case when $F \subset L_{\text {loc }}^{1}$, and then we will present the modification needed for the general case.

In spite of appearances, the theorem is essentially local, and is readily applicable to more general locally-convex spaces than the SCBS. For such spaces, 
one defines an analytic capacity associated to each seminorm, and the affineequivalence property involves pairs of such capacities in an obvious way. See section 4 below.

For quite a number of Beurling-invariant SCBS the result is already known, because the capacity has been characterised up to bounded equivalence in realvariable terms. For $0<\alpha<1$, the $\bar{\partial}$-Lip $\alpha$-cap is comparable to $(1+$ $\alpha$ )-dimensional Hausdorff content and the $\bar{\partial}$-lip $\alpha$-cap is comparable to lower $(1+\alpha)$-dimensional Hausdorff content [O' Farrell]. The $\bar{\partial}$-Lip1-cap is comparable to area measure [Hrushchev]. For $2<p<+\infty$, the $\bar{\partial}-L^{p}$-cap is an extremal length [Hedberg, Bagby]. The $\bar{\partial}$-BMO-cap is comparable to 1 dimensional Hausdorff content [Kaufmann]. An example of a space for which the result is new is ZC, the Zygmund class. The $\bar{\partial}-\mathrm{ZC}$-cap is not yet understood. The present theorem tells us that at least its null-sets form an affine-invariant class.

We will have occasion to make use of $\langle\phi, f\rangle$ in some situations where $f \in$ $\mathrm{C}_{\mathrm{cs}}^{\infty \prime}$ and $\phi \notin \mathrm{C}_{\mathrm{cs}}^{\infty}$. Specifically, we will use it when $\phi \in L_{\mathrm{loc}}^{1}, f \in \mathrm{C}_{\mathrm{cs}}^{\infty \prime}$, and $\phi$ is $\mathrm{C}^{\infty}$ on a neighbourhood $N$ of the singular support $S$ of $f$. The meaning we attach to $\langle\phi, f\rangle$ in this context is as follows. Choose any $\chi \in \mathrm{C}^{\infty}$ with spt $\chi \subset N$ and $\chi \equiv 1$ on $S$. Then $\phi \chi \in \mathrm{C}_{\mathrm{cs}}^{\infty}$, so $\langle\phi \chi, f\rangle$ makes sense, and $\phi(1-\chi) f \in L^{1}$, so $\int \phi(1-\chi) f d x d y$ makes sense. We define

$$
\langle\phi, f\rangle=\langle\phi \chi, f\rangle+\int \phi(1-\chi) f d x d y .
$$

It is readily seen that the result does not depend on the choice of $\chi$. We refer to $\langle\phi, f\rangle$ as the 'formal integral', and use the notation $\int \phi f d x d y$ for it when there is no danger of confusion.

\section{Proof of the Main Theorem}

The proof proceeds in steps. Throughout, we fix the Beurling-invariant SCBS, $F$, and we abbreviate

$$
\gamma(E)=\bar{\partial}-F-\operatorname{cap}(E) .
$$

We assume that $\gamma(E)>0$ for some compact set $E$, since otherwise the result is trivially true.

1. First, we define $\kappa(K, A)$ to be the least constant-possibly $+\infty$, a priori-such that

$$
\gamma(T E) \leq \kappa \gamma(E)
$$

whenever $E \subset K$ and $T \in A$. It is enough for us to prove that $\kappa(K, A)<+\infty$ whenever $K \subset \mathbf{C}$ is compact and $A$ is a compact subset of the affine group Aff.

2. For $A, B \subset$ Aff, let

$$
A B=\{S \circ T: S \in A, T \in B\},
$$

and define $A^{n}$ inductively by $A^{n+1}=A A^{n}$.

Let $R$ denote complex conjugation:

$$
R(x+i y)=x-i y .
$$

Let $B_{t}$ denote the unit ball of the group of translations:

$$
B_{t}=\{z \mapsto z+a: a \in \mathbf{C},|a| \leq 1\} .
$$

The following lemma is well known, and not hard to prove. 
Lemma 1. Let $U$ be any neighbourhood of the identity in $G L(2, \mathbf{R})$ and let $A$ be any compact subset of Aff. Then there exists $n \in \mathbf{N}$ such that

$$
A \subset\{1, R\} \cdot B_{t}^{n} \cdot U^{n} \text {. }
$$

3. Next, we observe that

$$
\begin{aligned}
\kappa(K, A B) & \leq \kappa(T K, A) \cdot \kappa(K, B), \\
\kappa\left(K, A^{n}\right) & \leq \kappa(K, A) \cdot \kappa(T K, A) \cdots \kappa\left(T^{n-1} K, A\right), \\
\kappa\left(K,\{1, R\} B_{t}^{n} U^{n}\right) & \leq \kappa\left(K^{\prime},\{R\}\right) \cdot \kappa\left(K^{\prime}, B_{t}^{n}\right) \cdot \kappa\left(K^{\prime}, U^{n}\right),
\end{aligned}
$$

provided $K^{\prime}$ contains $T K$ whenever $T \in\{1, R\} B_{t}^{n} U^{n}$ (Notice that $\kappa(K,\{1\}$ ) $=1$, and that $\kappa(K,\{R\}) \geq 1$. The latter property follows from the inequalities

$$
\gamma(E) \leq \kappa(K,\{R\}) \gamma(R E) \leq \kappa(K,\{R\})^{2} \gamma(E),
$$

since there exists some set $E$ having positive $\gamma(E)$.)

Thus it suffices to show that when $K$ is compact we have

$$
\kappa(K,\{R\})<+\infty, \quad \kappa\left(K, B_{t}\right)<+\infty,
$$

and that for some neighborhood $U$ of 1 in GL,

$$
\kappa(K, U)<+\infty \text {. }
$$

Lemma 2. $\kappa(K,\{R\})<+\infty$ whenever $K \subset \mathbf{C}$ is compact.

Proof. Fix $E$ compact, $E \subset K$. Let $f$ be $\bar{\partial}-F$-admissible for $R(E)$. Let $g=\overline{f \circ R}$. Then $g$ is analytic on $\mathbf{C} \sim E$, and

$$
\|g\|_{F}=\|f \circ R\|_{F} \leq \kappa_{1}\|f\|_{F}
$$

for some constant $\kappa_{1}$ independent of $f$, since $R$ acts continuously on $F$. Thus $g / \kappa_{1}$ is $\bar{\partial}-F$-admissible for $E$, so

$$
\gamma(E) \geq \frac{\left|g^{\prime}(\infty)\right|}{\kappa_{1}}=\frac{\left|f^{\prime}(\infty)\right|}{\kappa_{1}} .
$$

Taking the sup over all admissible $f$, we get

$$
\gamma(E) \geq \frac{\gamma(R E)}{\kappa_{1}},
$$

as required.

We remark that this result does not require the hypothesis of Beurlinginvariance on $F$, and that the bound obtained is independent of $K$. These features continue for the next series of lemmas, until we reach the final step in Lemma 7 below.

Lemma 3. $\kappa\left(K, B_{t}\right)<+\infty$ whenever $K \subset \mathbf{C}$ is compact.

Proof. Since $F$ is an SCBS and $B_{t}$ is compact, there exists a constant $\kappa_{2}>0$ such that

whenever $T \in B_{t}$.

$$
\|f \circ T\| \leq \kappa_{2}\|f\|_{F}
$$

Fix $E \subset K, E$ compact, and $T \in B_{t}$. If $f$ is $\bar{\partial}-F$-admissible for $T E$, then defining $g=f \circ T$, we see that $g$ is analytic off $E$, and

$$
\|g\|_{F} \leq \kappa_{2}\|f\|_{F} \leq 1
$$


so $g / \kappa_{2}$ is $\bar{\partial}-F$-admissible for $E$. As in the previous proof, we obtain

$$
\gamma(E) \geq \frac{\gamma(T E)}{\kappa_{2}}
$$

as required.

4. It remains to show that for some neighbourhood $U$ of 1 in GL, we have $\kappa(K, U)<+\infty$ for each compact $K \subset \mathbf{C}$.

Now the general element of $G L$ is a transformation of the form

$$
\left\{\begin{array}{l}
u=a x+b y, \\
v=c x+d y
\end{array}\right.
$$

and may be written as the composition of a dilation, a rotation, and an invertible map

$$
\left\{\begin{array}{l}
u=x, \\
v=c x+d y .
\end{array}\right.
$$

The latter may be written as the composition of a map of the form

$$
\left\{\begin{array}{l}
u=x, \\
v=y+a x
\end{array}\right.
$$

and an invertible map of the form

$$
\left\{\begin{array}{l}
u=x \\
v=a y .
\end{array}\right.
$$

Putting these observations together, and paying attention to bounds, we obtain the following. Let

$$
\begin{aligned}
O_{2} & =\left\{z \mapsto e^{i \alpha} z: \alpha \in \mathbf{R}\right\}, \\
D & =\{z \mapsto a z: a \in \mathbf{R} \text { and } 1 / 2 \leq a \leq 2\}, \\
G_{\varepsilon} & =\{(x, y) \mapsto(x, y+b x): b \in \mathbf{R},|b| \leq \varepsilon\}, \\
H_{\varepsilon} & =\{(x, y) \mapsto(x,(1+a) y): a \in \mathbf{R},|a| \leq \varepsilon\} .
\end{aligned}
$$

Lemma 4. Let $\varepsilon>0$ be given. The set of maps of the form $T_{1} T_{2} T_{3} T_{4}$, where $T_{1} \in O_{2}, T_{2} \in D, T_{3} \in G_{\varepsilon}$ and $T_{4} \in H_{\varepsilon}$, is a neighborhood of the identity in $G L(2, \mathbf{R})$.

5. It remains to show that

$$
\kappa(K, D)<+\infty, \quad \kappa\left(K, O_{2}\right)<+\infty,
$$

and that there exists $\varepsilon>0$ such that

$$
\kappa\left(K, G_{\varepsilon}\right)<+\infty \text { and } \kappa\left(K, H_{\varepsilon}\right)<+\infty .
$$

Note that in fact if these hold for some $\varepsilon>0$, then they hold for all $\varepsilon>0$, because

$$
\left(G_{\varepsilon}\right)^{n}=G_{n \varepsilon}, \quad\left(H_{\varepsilon}\right)^{n}=H_{\left((1+\varepsilon)^{n}-1\right)} .
$$

Lemma 5. $\kappa(K, D)<+\infty$ whenever $K$ is compact in $\mathbf{C}$.

Proof. Since $D$ is compact in Aff and $F$ is a SCBS, there exists $\kappa_{3}>0$ such that

$$
\|f \circ T\|_{F} \leq \kappa_{3} \cdot\|f\|_{F}
$$


whenever $f \in F$ and $T \in D$. Also, composition with dilations preserves analyticity, so the proof of this lemma goes through in exactly the same way as Lemma 3.

Lemma 6. $\kappa\left(K, O_{2}\right)<+\infty$ whenever $K$ is compact in $\mathbf{C}$.

Proof. This works in the same way as the previous lemma, since $O_{2}$ is compact in Aff and composition with rotations preserves analyticity.

6. This brings us to the estimate for $G_{\varepsilon}$ and $H_{\varepsilon}$. This is the subject of the next lemma, which is the core of the theorem, and is the only one that needs the Beurling invariance of $F$.

For real $\varepsilon$ we define the affine maps $S_{\varepsilon}$ and $T_{\varepsilon}$ by $S_{\varepsilon}(x, y)=(x, y+\varepsilon x)$ whenever $(x, y) \in \mathbf{C}$, and $T_{\varepsilon}(x, y)=(x,(1+\varepsilon) y)$ whenever $(x, y) \in \mathbf{C}$.

Lemma 7. Let $K \subset \mathrm{C}$ be compact. There exists $\varepsilon_{0}>0$ and $\kappa \geq 1$ such that if $|\varepsilon|<\varepsilon_{0}$, then

and

$$
\gamma\left(S_{\varepsilon} E\right) \leq \kappa \cdot \gamma(E)
$$

$$
\gamma\left(T_{\varepsilon} E\right) \leq \kappa \cdot \gamma(E)
$$

whenever $E$ is a compact subset of $K$.

Proof. The two estimates are very similar in proof. We will just prove the estimate for $T_{\varepsilon}$.

In the interest of clarity, we will first show how to prove the result on the additional hypothesis that $F \hookrightarrow L_{\text {loc }}^{1}$. Then we will show how to modify the proof to cover the general case.

We abbreviate $T_{\varepsilon}$ to $T$, to keep the formulas tidy. We only consider $\varepsilon$ with $|\varepsilon|<1$.

Fix $E$ compact, $E \subset K$.

Let $f$ be $\bar{\partial}-F$-admissible for $T E$, and suppose that $f^{\prime}(\infty)$ is real and greater than $\gamma(T E) / 2$. Then for large $|z|$ we have

$$
f(z)=\frac{a_{1}}{z}+\frac{a_{2}}{z^{2}}+\cdots
$$

and $a_{1}=f^{\prime}(\infty)$. So

$$
f(z) \sim \frac{f^{\prime}(\infty)}{z}, \quad f^{\prime}(z) \sim-\frac{f^{\prime}(\infty)}{z^{2}} .
$$

Fix $R>0$ large enough so that $|z| \leq R$ whenever $z \in T E$, and so that

$$
|f(z)| \leq \frac{2 \gamma(T E)}{|z|}
$$

and

whenever $|z|>R$.

$$
\left|f^{\prime}(z)\right| \leq \frac{2 \gamma(T E)}{|z|^{2}}
$$

Note that $R$ may depend on $E$ via $f$, and not just on $K$ and $A$.

We shall use const to stand for various constants that depend only on $F$ and $K$. We use $\lambda(R)$ to denote constants that depend on $F, K$, and on $R$.

We abbreviate

$$
\partial=\frac{\partial}{\partial z}=\frac{1}{2}\left(\frac{\partial}{\partial x}-i \frac{\partial}{\partial y}\right), \quad \bar{\partial}=\frac{\partial}{\partial \bar{z}}=\frac{1}{2}\left(\frac{\partial}{\partial x}+i \frac{\partial}{\partial y}\right) .
$$


Let $g=f \circ T$. A calculation yields

$$
\bar{\partial} g=\left(1+\frac{\varepsilon}{2}\right)(\bar{\partial} f) \circ T-\frac{\varepsilon}{2}(\partial f) \circ T,
$$

so that

$$
(\bar{\partial} g)(z)=-\frac{\varepsilon}{2} f^{\prime}(T(z))
$$

off $E$. (The corresponding calculation for $h=f \circ S$ yields

$$
\bar{\partial} g=\left(1+\frac{\varepsilon}{2}\right)(\bar{\partial} f) \circ T+\frac{\varepsilon}{2}(\partial f) \circ T .
$$

This is the only difference between the two cases.)

Let $g_{1}=\varepsilon \cdot C((\partial f) \circ T)$, where, as before, $C$ denotes the Cauchy transform. Let $g_{2}=g-g_{1}$. Note that $g_{2}$ is analytic on $\mathbf{C} \sim E$, and vanishes at $\infty$.

Suppose for a moment that $f$ is smooth. We then compute (making use of the fact that

$$
\frac{\partial}{\partial w}\left(\frac{1}{T^{-1}(z-w)}\right)=-\frac{\partial T^{-1}(w) / \partial w}{(L(z-w))^{2}}
$$

and

$$
\frac{\partial T^{-1}(w)}{\partial w}=\frac{1}{2}\left(\frac{1+\varepsilon-i}{1+\varepsilon}\right)
$$

is constant (independent of $w)$ ):

$$
\begin{aligned}
g_{1}(z) & =\frac{\varepsilon}{2 \pi} \int_{\mathbf{C}} \frac{1}{z-\zeta} \cdot(\partial f)(T(\zeta)) d \xi d \eta \quad(\text { where } \zeta=\xi+i \eta) \\
& =\frac{\varepsilon}{2 \pi|T|} \int_{\mathbf{C}} \frac{1}{z-T^{-1} w} \cdot \frac{\partial f(w)}{\partial w} d u d v \\
& \quad(\text { where } w=u+i v \text { and }|T| \text { is the determinant of } T) \\
& =\frac{-\varepsilon}{2 \pi|T|} \int_{\mathbf{C}} \frac{\partial}{\partial w}\left(\frac{1}{z-T^{-1} w}\right) f(w) d u d v, \\
g_{1}\left(T^{-1} z\right)= & \frac{(1+\varepsilon-i) \varepsilon}{2(1+\varepsilon)^{2}} \int_{C} \frac{f(w)}{(T-1(z-w))^{2}} d u d v, \\
g_{1}(z)= & \frac{(1+\varepsilon-i) \varepsilon}{2(1+\varepsilon)} \int_{\mathbf{C}} \frac{f(T(\zeta))}{(z-\zeta)^{2}} d \xi d \eta \\
= & \frac{(1+\varepsilon-i) \varepsilon}{2(1+\varepsilon)} B(f \circ T)(z) .
\end{aligned}
$$

Now once such a formula is true for smooth $f$, it is also true for general distributions. Thus for $|\varepsilon| \leq \frac{1}{2}$, we have

$$
\left\|g_{1}\right\|_{F} \leq 3 \cdot|\varepsilon| \cdot\|B(f \circ T)\|_{F} \leq \text { const } \cdot \varepsilon \cdot\|f\|_{F} .
$$

Thus

$$
\left\|g_{2}\right\|_{F} \leq\|g\|_{F}+\left\|g_{1}\right\|_{F} \leq \mathrm{const}
$$

and thus $g_{2} /$ const is a $\bar{\partial}-F$-admissible function for $E$.

We proceed to use limits along the positive $x$-axis to evaluate and estimate

$$
g_{2}^{\prime}(\infty)=\lim _{x \uparrow+\infty} x g(x)-\lim _{x \uparrow+\infty} x g_{1}(x) \text {. }
$$

We have

$$
\lim _{x \uparrow+\infty} x g(x)=\lim _{x \uparrow+\infty} x f(x)=f^{\prime}(\infty)>\gamma(T E) / 2
$$


Also

We split the integral

$$
\lim _{x \uparrow+\infty} x g_{1}(x)=\lim _{x \uparrow+\infty} \text { const } \cdot \varepsilon \cdot x \int \frac{f(w) d u d v}{\left(x-T^{-1} w\right)^{2}} .
$$

$$
I=\int \frac{f(w) d u d v}{\left(x-T^{-1} w\right)^{2}}
$$

into the integrals over three regions:

$I_{1}$ over $\left\{w:\left|T^{-1} w\right|<x / 2\right\}$,

$I_{2}$ over $\left\{w:\left|x-T^{-1} w\right|<x / 2\right\}$, and

$I_{3}$ over $\mathbf{C} \sim\left(I_{1} \cup I_{2}\right)$.

We assume $x$ is (much) larger than $2 R\|T\|$.

Since $F \hookrightarrow L_{\text {loc }}^{1}$, we have

$$
\int_{|z| \leq 2 R}|f(z)| d x d y \leq \lambda(R) \cdot\|f\|_{F} \leq \lambda(R)
$$

(following our convention, the constant $\lambda(R)$ depends on $F$ and $R$ ).

We split the integral $I_{1}$ into the integral

$I_{11}$ over $\left|T^{-1} w\right|<2 R\|T\|$ and the integral

$I_{12}$ over $2 R\|T\| \leq\left|T^{-1} w\right|<x / 2$, and we obtain

$$
I_{11} \leq \frac{4}{x^{2}} \int_{|z| \leq 2 R\|T\|}|f(z)| d x d y \leq \frac{\lambda(R)}{x^{2}}
$$

and

so

$$
I_{12} \leq \int_{0}^{2 \pi} \int_{2 R\|T\|}^{x / 2} \frac{\text { const } \cdot \gamma(T E) \cdot r d r d \theta}{r x^{2}} \leq \frac{\text { const } \cdot \gamma(T E)}{x}
$$

o

$$
\left|I_{1}\right| \leq \frac{\lambda(R)}{x^{2}}+\frac{\text { const } \cdot \gamma(T E)}{x}
$$

Thus for large $x$ we have

$$
\left|I_{1}\right| \leq \frac{\text { const } \cdot \gamma(T E)}{x}
$$

To estimate $I_{2}$, we observe that

$$
\begin{aligned}
I_{2} & =|T| \int_{|x-\omega|<x / 2} \frac{f(T(\omega))}{(x-\omega)^{2}} d \mu d \nu \\
\left|I_{2}\right| & \leq|T| \int_{|x-\omega|<x / 2} \frac{f(T(\omega))-f(T(x))}{(x-\omega)^{2}} \mid d \mu d \nu \\
& \leq|T| \int_{|x-\omega|<x / 2} \frac{\sup _{[x, \omega]}\left|f^{\prime}\right|}{|x-\omega|} d \mu d \nu \\
& \leq \text { const } \cdot \gamma(T E) \cdot|T| \int_{0}^{2 \pi} \int_{0}^{x / 2} \frac{1}{x^{2}} d r d \theta \\
& \leq \frac{\text { const } \cdot \gamma(T E) \cdot|T|}{x}
\end{aligned}
$$

since $\left|f^{\prime}(w)\right| \leq$ const $\cdot \gamma(T E) / x^{2}$ outside $\left|T^{-1} w\right| \leq x / 2$. 
Finally, if $x$ is large and $w \in \mathbf{C} \sim\left(I_{1} \cup I_{2}\right)$, then

$$
|f(w)| \leq \frac{\text { const } \cdot \gamma(T E)}{\left|x-T^{-1} w\right|}
$$

so

$$
\begin{aligned}
\left|I_{3}\right| & \leq \int_{\mathbf{C} \sim\left(I_{1} \cup I_{2}\right)} \frac{\text { const } \cdot \gamma(T E)}{\left|x-T^{-1} w\right|^{3}} d u d v \\
& \leq \int_{0}^{2 \pi} \int_{x / 2}^{\infty} \frac{\text { const } \cdot \gamma(T E)}{r^{2}} d r d \theta \\
& =\frac{\text { const } \cdot \gamma(T E)}{x} .
\end{aligned}
$$

Combining these estimates, we get

$$
\left|x g_{1}(x)\right| \leq \text { const } \cdot|\varepsilon| \cdot \gamma(T E) \leq \text { const } \cdot \varepsilon_{0} \cdot \gamma(T E)
$$

for large $x$.

Provided $\varepsilon_{0}$ is small enough to make

$$
\text { const } \cdot \varepsilon_{0} \leq 1 / 4
$$

we have

$$
\left|\lim _{x \uparrow \infty} x g_{1}(x)\right| \leq \frac{\gamma(T E)}{4}
$$

so

so

$$
\left|g_{2}^{\prime}(\infty)\right| \geq \frac{\gamma(T E)}{2}-\frac{\gamma(T E)}{4}=\frac{\gamma(T E)}{4}
$$

$$
\gamma(E) \geq \frac{\gamma(T E)}{4\left\|g_{2}\right\|_{F}} \geq \text { const } \cdot \gamma(T E) .
$$

This concludes the proof of the main theorem in the case where $F \hookrightarrow L_{\text {loc }}^{1}$.

In order to prove the theorem in general, it is only necessary to modify the estimate of $I_{11}$, which must now be regarded as a formal integral, in the sense discussed in the introduction.

To show that $I_{11}$ is $O\left(\lambda(R) / x^{2}\right)$, choose $\chi \in \mathrm{C}_{\mathrm{cs}}^{\infty}$ such that $\chi=1$ near $E$ and spt $\chi \in\left\{\left|T^{-1} w\right|<2 R\|T\|\right\}$. The choice of $\chi$ may be made independently of $f$. There is no problem estimating $\int(1-\chi(w)) f(w) /\left(x-T^{-1} w\right)^{2} d u d v$, so it reduces to estimating

$$
\left\langle\frac{\chi}{\left(x-T^{-1} w\right)^{2}}, f\right\rangle \text {. }
$$

Since $F \hookrightarrow \mathrm{C}_{\mathrm{cs}}^{\infty \prime}$, this expression is dominated by an expression of the form

$$
\|f\|_{F} \cdot \text { const } \cdot \sum_{k=0}^{r}\left\|D^{k}\left(\frac{\chi}{\left(x-T^{-1} w\right)^{2}}\right)\right\|_{L^{\infty}},
$$

where $r$ is some natural number. But a simple application of Leibnitz's rule shows that

so we are done.

$$
\left|D^{k}\left(\frac{\chi}{\left(x-T^{-1} w\right)^{2}}\right)\right| \leq \frac{c_{k}}{x^{2}}
$$




\section{NON-BANACH SPACES}

In conclusion, we indicate how the result may be extended to a wider class of spaces, the symmetric concrete spaces (SCS). These are complete locally-convex topological vector spaces $F$ of distributions such that the inclusions

$$
\mathrm{C}_{\mathrm{cs}}^{\infty} \hookrightarrow F \hookrightarrow \mathrm{C}_{\mathrm{cs}}^{\infty \prime}
$$

are continuous, $F$ is a topological $\mathrm{C}_{\mathrm{cs}}^{\infty}$-module with respect to pointwise multiplication, $f \mapsto \bar{f}$ is an endomorphism of $F$, and compact sets of invertible affine transformations of $\mathbf{C}$ act equicontinuously on $F$ by composition. The spaces $\mathrm{C}_{\mathrm{cs}}^{\infty}$ and $\mathrm{C}^{\infty}$ are non-Banach examples. Given any $\operatorname{SCS} F$, one forms new SCS

$$
\begin{aligned}
F_{\mathrm{loc}} & =\left\{f \in \mathrm{C}_{\mathrm{cs}}^{\infty \prime}: \phi \cdot f \in F, \forall \phi \in \mathrm{C}_{\mathrm{cs}}^{\infty}\right\}, \\
F_{\mathrm{cs}} & =\left\{f \cdot \phi: \phi \in \mathrm{C}_{\mathrm{cs}}^{\infty}\right\} .
\end{aligned}
$$

Two SCS $F$ and $G$ are said to be locally-equivalent if $F_{\text {loc }}=G_{\text {loc }}$.

For SCBS $F$, one also defines

$$
\|f\|_{F(K)}=\inf \left\{\|g\|_{F}: g=f \text { near } K\right\}
$$

whenever $f \in F_{\text {loc }}$ and $K$ is compact. The seminorms $\|f\|_{F(K)}$ define the topology of $F_{\text {loc }}$. The space

$$
F_{\infty}=\left\{f \in F_{\mathrm{loc}}: \sup _{a \in \mathrm{C}}\|f\|_{F(\mathrm{~B}(a, 1))}<+\infty\right\}
$$

is locally-equivalent to $F$ but is somewhat better-behaved in general. Translation acts isometrically on $F_{\infty}$, and it has other nice properties.

We say that $F$ is $B$-invariant if $B$ maps $F \cap$ EL3 (with the relative $F$ topology, not the intersection topology) continuously into $F$.

This can be quite a stringent condition, and some weaker conditions are useful:

We say that $F$ is locally $B$-invariant if $B$ maps $F \cap$ EL3 continuously into $F_{\text {loc }}$. $F$.

We say that $F$ is co-locally $B$-invariant if $B$ maps $F_{\mathrm{cs}}$ continuously into

We say that $F$ is bi-locally $B$-invariant if $B$ maps $F_{\mathrm{cs}}$ continuously into $F_{\text {loc }}$. This is the weakest of the four conditions.

An analytic capacity may be associated in an obvious way to each $[0,+\infty]-$ valued seminorm $s$ on $\mathrm{C}_{\mathrm{cs}}^{\infty \prime}$ :

$$
\bar{\partial}-s-\operatorname{cap}(E)=\sup \left\{\left|f^{\prime}(\infty)\right|\right\}
$$

where $f$ runs over all $f \in F$ that are analytic off $E$, vanish at $\infty$, and have $s(f) \leq 1$. We say that an SCS $F$ has the affine-equivalence property for analytic capacities if given $K$ compact in $\mathbf{C}, A$ compact in Aff, and a continuous seminorm $s$ on $F$, there exists a continuous seminorm $t$ on $F$ such that

$$
\bar{\partial}-s-\operatorname{cap}(T E) \leq \bar{\partial}-t-\operatorname{cap}(E)
$$

whenever $E \subset K$ and $T \in A$. With this generalisation of the concept, it is straightforward to prove the following generalisation of the theorem: 
Theorem'. If $F$ is a SCS and $B: F \cap \mathrm{EL} 3 \rightarrow F$ is continuous in $F$-topology, then $F$ has the affine-equivalence property for analytic capacities.

With a little more effort, one can show that if $F$ is a local SCS, i.e. $F=F_{\text {loc }}$, and $F$ is bilocally- $B$-invariant, then $F$ has the affine-equivalence property for analytic capacities. One can also show that if $F$ is a SCBS and $F$ is bilocally$B$-invariant, then $F_{\infty}$ has the affine-equivalence property for analytic capacities. Details of this will appear elsewhere.

The space $L^{2}$ illustrates the importance of $F_{\infty}$. The $\bar{\partial}-L^{2}$-cap is identically zero, and so cannot be used to any effect. The $\bar{\partial}-L_{\infty}^{2}$-cap is related to logarithmic capacity [Hedberg] and provides a workable substitute. A similar story applies to $L^{p}$ for $1 \leq p<2$.

\section{REFERENCES}

L. V. Ahlfors, Bounded analytic functions, Duke Math. J. 14 (1947), 1-11.

T. Bagby, $L_{p}$ approximation by analytic functions, J. Approx. Theory 5 (1972), 401-404.

E. M. Stein, Singular integrals and differentiability properties of functions, Princeton Univ. Press, Princeton, NJ, 1970.

L. I. Hedberg, Approximation in the mean by analytic functions, Trans. Amer. Math. Soc. 163 (1972), 157-171.

S. V. Hrushchev, Simple proof of a theorem on removable singularities for analytic functions, LOMI Notes, Leningrad, 1975.

A. G. O'Farrell, Hausdorff content and rational approximation in fractional Lipschitz norms, Trans. Amer. Math. Soc. 228 (1977), 187-206.

T. Gamelin, Uniform algebras, Prentice-Hall, Englewood Cliffs, NJ, 1970.

R. Kaufmann, Hausdorff measure, BMO, and analytic functions, Pacific J. Math. 102 (1982), 369-371.

Department of Mathematics, St. Patrick's College, Maynooth, County Kildare, IRELAND

E-mail address: tdowling@maths.may.ie

E-mail address: aof@maths.may.ie 